\title{
Correspondence
}

British Heart fournal, 1977, 39, 116

\section{Rules for diagnosis of arterioventricular discordances and spatial identification of ventricles}

Sir,

I have not written before commenting on Macartney et al.'s criticism of my paper entitled 'Rules for diagnosis of arterioventricular discordances and spatial identification of ventricles: crossed great arteries and transposition of the great arteries', because I had not yet received the issue of the British Heart fournal containing both publications $(38,323,341)$. Though some of the objections of Macartney and his colleagues to my papers are explicitly or implicitly answered in my publications, I would like to refute some of their most controversial statements.

The hypotheses of differential growth and of differential absorption of the conus are based on the supposed 'fact' that when both coni appear during normal embryonic development, the pulmonary conus is on the left side and the aortic conus on the right. Based on this false assumption, they then suggest that differential growth or absorption of the coni can explain the different truncoconal morphologies, namely crossed great arteries, transposition of the great arteries, and great arteries situated side-byside. Both hypotheses are wrong because, from the moment of appearance of both coni during embryonic development, the pulmonary conus is anterior and right-sided while the aortic conus is posterior and left-sided, as may be seen in the photographs in my paper. Furthermore, the methodology used for the study of the differential absorption of the conus is unsound, since it is impossible to measure a segment of the heart whose limits are unknown; even if those limits were known, measurements would have to be made in the same embryo at different stages of development to determine if there had been growth or absorption, because embryos of the same age may not be the same size.

Even though our findings give support to the hypotheses proposed by ourselves and by Van Mierop, we believe that instead of continuing with unproductive discussions about the validity of different hypotheses, more embryological work should be done to elucidate the basic developmental processes that occur in the formation of the normal heart. When we understand these developmental processes, it will then be possible to explain the morphogenesis of the congenital malformations of the heart, and also to produce some cardiac malformations experimentally by specific alterations of processes involved in the normal development of the heart.

Since one of the authors of the Editorial is an embryologist, he should realise that it is still unknown which of the basic processes of development (cellular growth, cellular death, cell differentiation, and cell movements) are involved in the incorporation of the posterior conus into the left ventricle, and that Pexieder in his study does not state that cellular death is the causal factor.

Following the established practice of referring to atrioventricular concordance or discordance to indicate whether the type of bulboventricular loop corresponds to visceroatrial situs or not, we have used the designations arterioventricular concordance and discordance to indicate whether the type of bulboventricular loop corresponds with the spatial orientation of the truncus septum or not.

We have never stated that the angiocardiographic identification of the ventricles by the relations of the great arteries to one another and to the infundibula, as we have proposed, and their identification from their intrinsic features are mutually exclusive; on the contrary, these methods complement one another.

When referring to atrioventricular concordance or discordance, we prefer the term 'relation' to 'connection' since it has a more universal meaning; thus, when there is an anatomically right atrium to the right and an anatomically right ventricle to the right, whether or not there is atresia of the right atrioventricular orifice, there is a concordant atrioventricular relation; but when the right atrioventricular valve is atretic, there is no atrioventricular connection.

Maria Victoria de la Cruz, Instituto Venezolano de Cardiologia, Apartado 59019, Caracas 104, Venezuela. 